\title{
Zinc Oxide Nanoparticles Prepared by the Reaction of Zinc Metal with Ethanol
}

\author{
M. A. Shah and M. Al-Shahry ${ }^{1}$ \\ Department of Physics, and ${ }^{1}$ Department of Chemistry, Faculty of \\ Science, King Khalid University, Abha, Saudi Arabia
}

\begin{abstract}
Zinc oxide nanoparticles have been prepared by a very simple reaction of zinc metal with ethanol at $200{ }^{\circ} \mathrm{C}$. The diameters of the nanoparticles range from 50-200 $\mathrm{nm}$ with an average diameter of $100 \mathrm{~nm}$. The reaction involves the cleavage of C-O bond of the alcohol, which occurs readily on zinc metal surface. Addition of ethylenediamine to the reaction yields nanorods, the amine acting as a shape controlling agent. A plausible mechanism is proposed for the formation of these nanostructures and is expected that this synthetic technique can be extended to obtain other metal oxides.
\end{abstract}

Keywords: Nanostructures, synthesis, ethanol, shape-controlling agent.

\section{Introduction}

The unique properties of nanomaterials have motivated the researchers to develop the simpler and inexpensive techniques to produce nanostructures of technologically important materials. Zinc oxide is particularly important for its wide range of applications. The wide band gap of $3.3 \mathrm{eV}$ and large excitonic binding energy of $60 \mathrm{meV}$ have made this material important both for scientific as well as industrial applications ${ }^{[1]}$. Moreover, the wide range of morphological diversity in the nanoregime has made this material a promising candidate in the field of nanotechnology and opened up new possibilities for the fabrication of high performance devices based on these nanostructures. Among the various nanoforms, one dimensional (1D) nanostructures such as nanorods, nanowires, nanotubes and nanopins have received considerable attention due to their potential interests for understanding

*Corresponding author: M.A.Shah, E.Mail: shah11nit@gmail.com, Fax: 00966-72290165 
fundamental physical concepts and for efficient field emission that has enormous commercial applications such as field emission flat panel displays, x-ray sources, parallel beam electron microscopy and vacuum microwave amplifiers ${ }^{[2-5]}$.

A number of synthetic routes have been employed to synthesis of $\mathrm{ZnO}$ nanoparticles, such as sol-gel chemistry, spray pyrolysis, physical vapour deposition, precipitation, solvothermal and hydrothermal method $^{[6-10]}$. Through evaporation and condensation process are favoured for their simplicity and high quality products, they are generally constrained by the requirement of high temperature and use of catalysts that can produce unintentional defects. Hence low temperature and versatile synthetic process are in need and are of great importance for the realization of devices based on these nanostructures.

A detailed study has revealed that interaction of alcohols with $\mathrm{Zn}$ metal has shown that $\mathrm{C}-\mathrm{O}$ bond of the alcohol is readily cleaved on zinc metal surface giving hydrocarbons and the oxide species on the metal surface. Recently we have reported the synthesis of $\mathrm{ZnO}$ nanorods by a simple reaction of zinc metal with water at moderate temperature without any additives but failed to produce nanoparticles ${ }^{[11]}$. In this work, we have successfully synthesized $\mathrm{ZnO}$ nanoparticles by a simple reaction of zinc metal with ethanol at $200^{\circ} \mathrm{C}$. The nanoparticles are not entirely spherical in shape and have diameters varying between 50 to $150 \mathrm{~nm}$, with an average diameter of $100 \mathrm{~nm}$. Addition of ethylenediamine to the reaction yields nanorods, thus the amine acting as a shape controlling agent. Detailed and systematic studies would be necessary to optimize the conditions for obtaining nanoparticles of desired dimensions. Earlier, under solvothermal conditions, $\mathrm{ZnO}$ nanorods have been obtained by reacting zinc acetate with $\mathrm{NaOH}$ in presence of polyvinylpyrrolidone (PVP) at $80^{\circ} \mathrm{C}$ in ethanol ${ }^{[12]}$.

\section{Experimental}

In a typical synthesis, $5 \mathrm{mg}$ of $\mathrm{Zn}$ metal powder was added to $10 \mathrm{ml}$ of ethanol. The reaction mixture was sonicated for about $20 \mathrm{~min}$, transferred into a stainless steel autoclave and sealed under inert conditions. The reaction mixture was heated slowly $\left(2^{\circ} \mathrm{C} / \mathrm{min}\right)$ to $200^{\circ} \mathrm{C}$ and maintained at this temperature for 24 and $48 \mathrm{~h}$ respectively. The resulting suspension was centrifuged to retrieve the product, washed and then finally vacuum dried. 
Zinc oxide nanorods could be prepared by the addition of $2 \mathrm{ml}$ ethylenediamine to a mixture of $10 \mathrm{mg}$ of zinc metal powder and $10 \mathrm{ml}$ of ethanol and heated to $200^{\circ} \mathrm{C}$.

Field emission scanning electron microscope (FESEM) images were recorded with a FEI NOVA NANOSEM 600 coupled with EDX. X-ray diffraction patterns of the samples were recorded with Siemens D 5005 diffractometer using $\mathrm{Cu} \mathrm{K \alpha}(\lambda=0.15141 \mathrm{~nm})$ radiation. Photoluminescence spectra were recorded with Perkin-Elmer model LS55 luminescence spectrometer.

\section{Results and Discussion}

Figure 1 shows various types of zinc oxide nanoparticles obtained by reaction of zinc metal powder with methanol at $200^{\circ} \mathrm{C}$ for 24 and $48 \mathrm{~h}$ respectively. Figure 1(a) shows the morphology of nanoparticles obtained after the reaction of zinc with water at $200^{\circ} \mathrm{C}$ for $24 \mathrm{~h}$. The nonoparticles are not entirely spherical in shape and have diameters varying between 50 to $150 \mathrm{~nm}$, with an average diameter of $100 \mathrm{~nm}$. Figure 1 (b) shows the morphology of nanoparticles obtained after the reaction of zinc with methanol at $200^{\circ} \mathrm{C}$ for $48 \mathrm{~h}$. In some regions we notice the big nanoparticles (average diameter $200 \mathrm{~nm}$ ) are surrounded by smaller nanoparticles. The effect of reaction time plays a marvelous role in the morphology of nanoparticles. The influence of reaction conditions on physical properties of synthesized nanoparticles as well as mechanism is yet to be investigated.
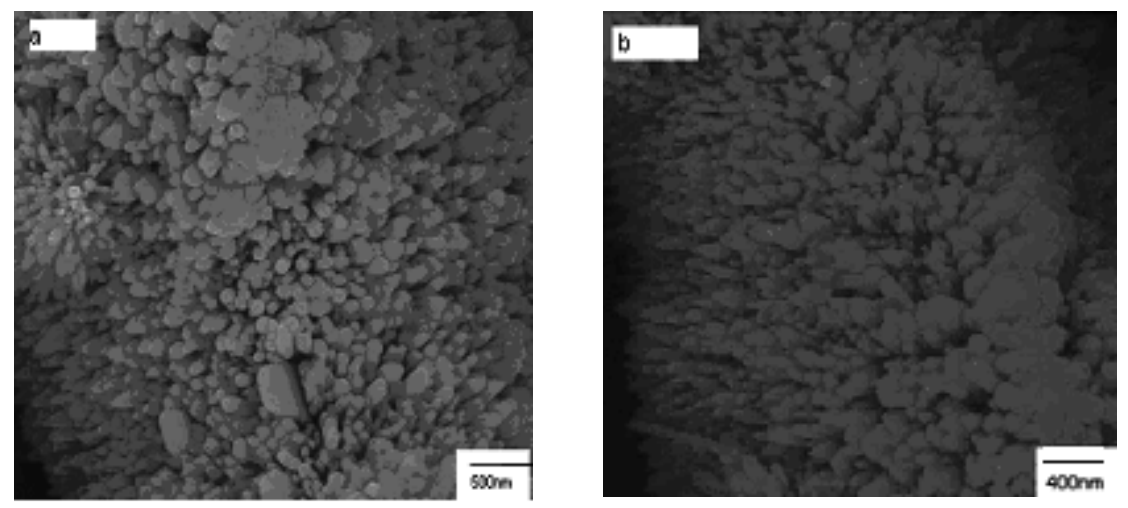

Fig. 1. The FESEM images of Nanoparticles of $\mathrm{ZnO}$ prepared by zinc metal with ethanol at $200^{\circ} \mathrm{C}$ for 24 and $48 \mathrm{~h}$ respectively. 
Addition of ethylenediamine to the reaction mixture of zinc metal powder and alcohol yields nanorods. Figure 2 ( $a$ and $b$ ) shows the FESEM images of $\mathrm{ZnO}$ nanorods obtained by addition of ethylenediamine to the mixture of zinc and ethanol at $200^{\circ} \mathrm{C}$ for 24 and $48 \mathrm{~h}$ respectively. The nanorods obtained with ethanol have an average diameter of $100 \mathrm{~nm}$ and lengths upto several micrometers. We also observe the occurrence of smaller $\mathrm{ZnO}$ nanorods with an average diameter of $50 \mathrm{~nm}$ and average length of $150 \mathrm{~nm}$. The effect of ethylenediamine to the ethanol acted as a shape-directing agent to the amines in agreement with the literature ${ }^{[13]}$.
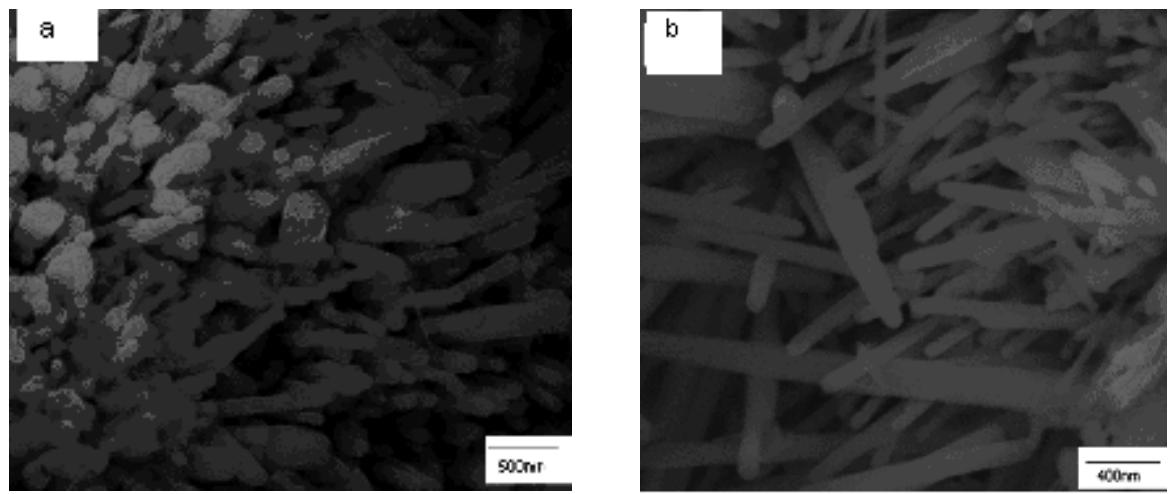

Fig. 2. The FESEM images of nanorods of $\mathrm{ZnO}$ prepared by addition of ethylenediamine to the mixture of zinc metal and ethanol at $200^{\circ} \mathrm{C}$ for 24 and $48 \mathrm{~h}$ respectively.

The EDX measurement on the nanoparticles and nanorods indicates that the nanostructures are composed of $\mathrm{Zn}$ and $\mathrm{O}$ as shown in Fig. 3. The molecular ratio of $\mathrm{Zn} / \mathrm{O}$ of the nanostructures, calculated from EDX and quantitative analysis data is close to that of bulk $\mathrm{ZnO}$. The XRD patterns of the $\mathrm{ZnO}$ nanoparticles could be indexed to the hexagonal wurtzite structure (space group: P6 ${ }_{3} \mathrm{mc} ; \mathrm{a}=0.3249 \mathrm{~nm}, \mathrm{c}=0.5206 \mathrm{~nm}$, JCPDS card No. 36-1451).

The photoluminescence (PL) methodology is generally adopted for investigating the radioactive recombination emission processes due to the excitation, defect and impurity levels in semiconductors. It is well known that there are two kinds of emission bands of UV and visible spectrum in $\mathrm{ZnO}$ crystals. The emission in the $\mathrm{UV}$ region is attributed to the recombination between electrons in conduction band and holes in valence band $^{[14]}$. The visible emission is related to the defects related deep level emission such as oxygen vacancies and $\mathrm{Zn}$ interstitials. Therefore, a 
study of PL property of $\mathrm{ZnO}$ can provide valuable information on the quality and purity of this material. Figure 4 shows room temperature PL spectrum of the $\mathrm{ZnO}$ nanoparticles shows a UV emission at $370 \mathrm{~nm}$ and broad bands centered at $440 \mathrm{~nm}, 460 \mathrm{~nm}, 480 \mathrm{~nm}$ and $485 \mathrm{~nm}$. The well known stronger and broader emission situated in the yellow green part of the visible spectrum is much lower, which suggests that the obtained $\mathrm{ZnO}$ nanoparticles are high pure and crystalline.

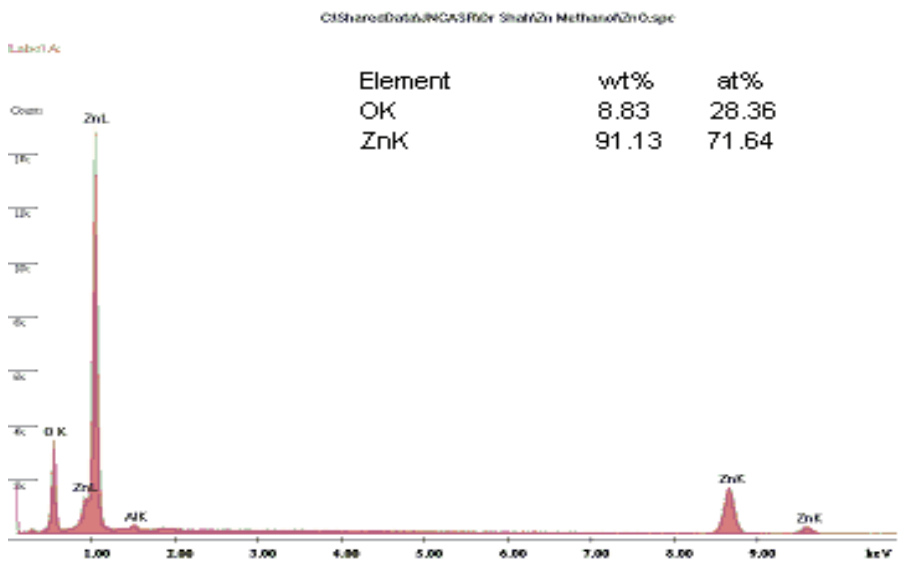

Fig. 3. The corresponding EDX analysis confirming the existence of all elements involved in sample preparation.

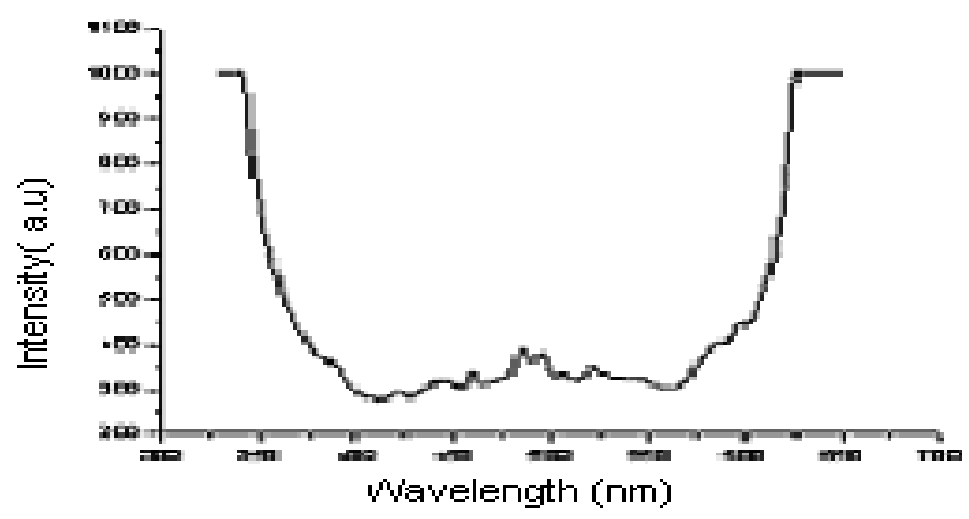

Fig. 4. Room temperature photoluminance spectra (excitation at $375 \mathrm{~nm}$ ) of $\mathrm{ZnO}$ nanoparticles. 
There are two well accepted mechanisms for the growth of one dimensional nanostructures, viz. the vapour-liquid-solid (VLS) and vapour-solid (VS) mechanism. The VLS mechanism is a catalyst assisted process, in which the metal catalyst particles act as a liquid forming agent. In the present work, VLS mechanism can be firstly excluded because catalysts are not used in our experiments. The growing temperature for the $\mathrm{ZnO}$ nanorods was just $300^{\circ} \mathrm{C}$. $\mathrm{Zn}$ vapour could not be present at such a temperature. The VS mechanism is not also appropriate for it. From our experiments and analysis, it suggests that the growing mechanism of $\mathrm{ZnO}$ nanorods may be simple diffusion process, but it still has large space to be studied.

\section{Conclusion}

We have presented a very simple approach for the synthesis of $\mathrm{ZnO}$ nanoparticles/nanorods by a simple reaction of zinc metal with alcohol. This is in accordance with the finding that the $\mathrm{C}-\mathrm{O}$ bond of alcohols is readily cleaved by $\mathrm{Zn}$ metal. The ethylenediamine acted as the director and the shape controlling agent. This facile, reproducible and effective low cost approach should promise us a future large scale synthesis of $\mathrm{ZnO}$ nanostructures for many applications in nanotechnology.

\section{Acknowledgement}

One of the authors (Dr. M.A. Shah) is thankful to World Bank for their financial support in procuring SEM.

\section{References}

[1] Klingshrin, C., Chem. Phys. Chem., 8: 782 (2007).

[2] Wang, Z.L., Mater. Today, 7: 26 (2004).

[3] Cui, Y., Wei, Q.Q., Park, H.K. and Libera, C.M., Science, 293: 1289 (2001).

[4] Baughman, R.H., Zakhidev, A.V. and Heer, W.A.D., Science, 279: 787 (2002).

[5] Senda, S., Sakai, M., Mizuta, Y., Kita, S. and Okuyama, F., Appl. Phys. Lett., 85: 5679 (2004).

[6] Shim, M. and Sionnest, P.G., J. Am. Chem. Soc., 123: 11651 (2001).

[7] Kamalasanan, M.N. and Chandra, S., Thin Solid Films, 288: 112 (1996).

[8] Muller, R., Madler, L. and Pratsinis, S.E., Chem. Eng. Sci., 58: 1969 (2003).

[9] Chakarbarti, S. and Chaudhari, G., Mater. Chem. Phys., 87: 196 (2004).

[10] Hari kumar, K.R. and Rao, C.N.R., J. Phys. Chem., B 103: 2445 (1999).

[11] Panchakarla, L.S., Shah, M.A., Govind Raj, A. and Rao, C.N.R., J. Solid Stat Chem., 180: 113106 (2007).

[12] Liu, B. and Zeng, H.C., J. Am. Chem. Soc., 125: 4430 (2003)

[13] Wang, C., et al., Mater. Lett., 59: 2867 (2005).

[14] Hu, J.Q. and Bando, Y., Appl. Phys. Lett., 82: 1401 (2003). 


\title{
الجسيمات النانونية لأكسبد الزنك المحضرة بو اسطة التفاعل بين فلز الزنك و الإيثانول
}

\author{
محمد أشرف شاه و مفرح سعيد الشهري

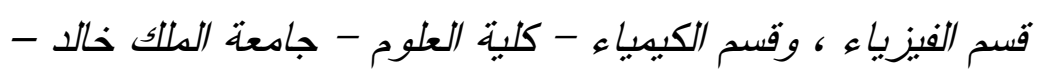 \\ المملكة العربية السعودية
}

السستخلص. نم تحضير الجسيمات النانونية لأكسيد الزنك بواســة

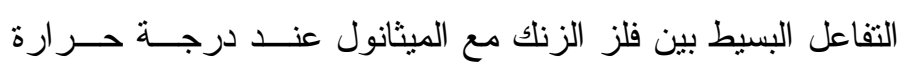

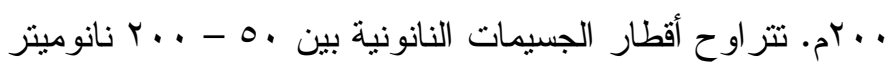

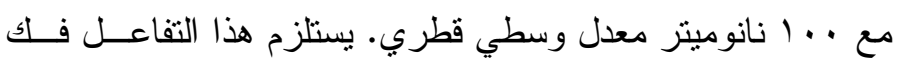

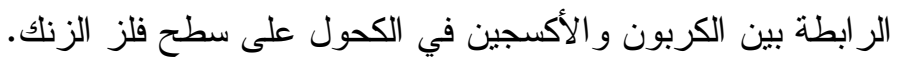

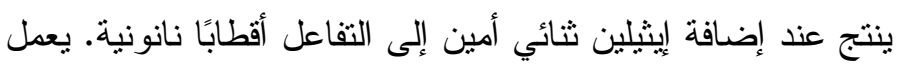

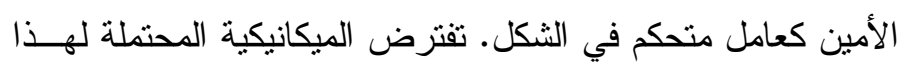

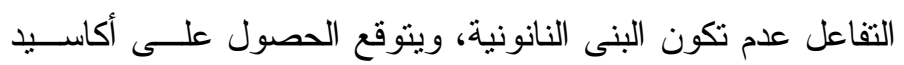
فلزية أخرى باستخدام هذه الطريقة البسيطة. 